\title{
Identifying research priorities on infections in older adults: proceedings of an interdisciplinary workshop Mark Loeb ${ }^{* 1,2,3}$, Kevin Brazil ${ }^{3}$, Pierre Durand ${ }^{4}$, Michael Gordon ${ }^{5}$, Paul Krueger ${ }^{3}$, David Lewis ${ }^{6}$, Lynne Lohfeld ${ }^{3}$, Allison McGeer ${ }^{7,8}$, Lindsay Nicolle ${ }^{9}$, Alexandra Papaioannou ${ }^{10}$ and Andrew E Simor ${ }^{8,11}$
}

Address: ${ }^{1}$ Hamilton Regional Laboratory Program, Mc Master University, Hamilton, Canada, ${ }^{2}$ Department of Pathology and Molecular Medicine,Mc Master University, Hamilton, Canada, ${ }^{3}$ Department of Clinical Epidemiology and Biostatistics, Mc Master University, Hamilton, Canada, ${ }^{4}$ Centre de Research du Centre Hospitalier affilie Universitaire de Quebec, Departement de Medicine, L'Universite de Laval, Quebec, Canada, ${ }^{5}$ Department of Geriatrics, Baycrest Centre for Geriatric Care, Department of Medicine, The University of Toronto, Toronto, Canada, ${ }^{6}$ Father Sean O'Sullivan Research Centre, St. Joseph's Hospital, Hamilton, and the Department of Sociology, McMaster University, Hamilton, Canada, ${ }^{7}$ Department of Microbiology, Mount Sinai Hospital, Toronto, Canada, ${ }^{8}$ Department of Laboratory Medicine and Pathobiology, University of Toronto, Toronto, Canada, ${ }^{9}$ Winnipeg Health Sciences Centre and the Department of Medicine, University of Manitoba, Winnipeg, Canada, ${ }^{10}$ Hamilton Health Sciences Corporation, Hamilton, Department of Medicine, McMaster University, Hamilton, Canada and ${ }^{11}$ Department of Microbiology, Sunnybrook and Women's College Health Sciences Centre, Toronto, Canada

E-mail: Mark Loeb* - loebm@mcmaster.ca; Kevin Brazil - brazilk@mcmaster.ca; Pierre Durand - pierre.Durand@msp.ulaval.ca; Michael Gordon - mgordan@baycrest.org; Paul Krueger - kruegerp@mcmaster.ca; David Lewis - Lewis_David/depts@email.stjosham.on.ca; Lynne Lohfeld - llohfeld@sjhh.guelph.on.ca; Allison McGeer - amcgeer@mtsinai.on.ca;

Lindsay Nicolle - nicolle@cc.umanitoba.ca; Alexandra Papaioannou - papaioannou@ hhsc.ca; Andrew E Simor - andrew.simor@swchsc.on.ca *Corresponding author

Published: 14 August 200 I

Received: 7 May 200I

BMC Geriatrics 200 I, I:I

Accepted: 14 August 200I

This article is available from: http://www.biomedcentral.com/I47I-23/8/I/I

(C) 200 I Loeb et al; licensee BioMed Central Ltd. Verbatim copying and redistribution of this article are permitted in any medium for any non-commercial purpose, provided this notice is preserved along with the article's original URL. For commercial use, contact info@biomedcentral.com

\begin{abstract}
Background: Infections pose a substantial burden to the health of older adults. In this report, we describe the proceedings of a workshop to formulate and prioritize research questions about infections in older adults using an interdisciplinary approach.
\end{abstract}

Methods: Researchers from four sectors (basic science, clinical sciences, health services and epidemiology/ determinants of health) and representatives from various Canadian local, provincial, and federal stakeholder groups were invited to a two-day workshop. Five multi-disciplinary groups and stakeholders from each of three healthcare settings (long term, acute care and community) discussed research priorities for each of the settings. Five to ten research questions were identified for each setting.

Results: The research questions proposed ranged from risk factors and outcomes for different infections to the effect of nutrition on infection and the role of alternative and complementary medicine in treating infections. Health service issues included barriers to immunization, prolongation of hospital length of stay by infection, use of care paths for managing infections, and decision-making in determining the site of care for individuals with infections. Clinical questions included risk factor assessment for infection, the effectiveness of preventative strategies, and technology evaluation. Epidemiologic issues included the challenge of achieving a better understanding of respiratory infections in the community and determining the prevalence of colonization with multi-resistant bacteria.

Conclusions: The questions are of direct relevance to researchers in a wide variety of fields. Bringing together a multi-disciplinary group of researchers to frame and prioritize research questions about aging is feasible, participants valued the opinions of people working in other areas. 


\section{Background}

Older adults consume a disproportionate amount of healthcare resources in Canada. Persons over the age of 65 years make up $12 \%$ of the general population, but account for $31 \%$ of acute hospital days and half of all hospital stays [1]. The proportion of older adults in Canada is expected to rise to $20 \%$ by 2021 [2], so meeting the future healthcare needs of this vulnerable population will be formidable. The provision of care for older adults with infectious diseases will be part of this challenge. The vast majority of excess deaths and hospitalizations due to respiratory infections occur in older adults, with more than 44,00o hospitalizations for pneumonia and influenza in people aged 65 and older in 1997 [1]. In Canada, as well as in the United States, older adults who live in long-term care facilities are at especially high risk of these respiratory infections, for which they are frequently transferred to hospital [3-6].

To improve the care of older adults with infections, a research agenda which incorporates a wide range of issues is needed. Questions about the basic biology, clinical science, delivery of health services, and broader determinants of infectious diseases in older adults need to be generated and prioritized. We believe that an interdisciplinary approach to identifying, prioritizing, and conducting research about infections in older adults will result in better health for this population. In the development of the Canadian Institutes of Health Research, Canada's new health research funding agency organized through a framework of 13 "virtual" institutes, Canada's three major federal funding agencies (Medical Research Council, National Science and Engineering Research Council, Social Sciences and Humanities Research Council) sponsored workshops in 1999 to foster collaboration among researchers from four disciplines: epidemiology/determinants of health, health services, clinical sciences, and basic science. This Tricouncil Workshop/ Networking Program was designed to promote research linkages that bridge the traditional boundaries of research activity supported by the councils individually. It supported multi-disciplinary workshops as well as the development of research agendas, collaborative networks and other similar initiatives to assist health research scientists to compete effectively for Canadian Institutes of Health Research funding opportunities. This was to be done by developing and strengthening research contacts, networks and groups on a specific health research theme, through exchanges of knowledge, insights and approaches among researchers, practitioners and users. It was also to test innovative ideas and approaches for tackling medium to long-term issues in health research in areas which require the collaboration of disciplines or faculties traditionally supported by at least two of the three granting councils.
In this report, we describe the proceedings of the workshop entitled "Identifying research priorities on infections in older adults", held in Hamilton, Ontario on April 13 and 14, 2000. The objective of this workshop was to formulate and prioritize research questions of infections in older adults using an interdisciplinary framework.

\section{Methods}

Participant and stakeholder lists were developed by a 12member committee and expanded through Internet searches of seniors-related web-sites and literature. Speakers for the workshop were selected based on their extensive published research and experience in each of the four research disciplines.

Invited participants were selected from each of four research disciplines: basic science, clinical sciences, health services and epidemiology/determinants of health. Participants included geriatricians, basic scientists, medical directors of long-term care facilities, nurse specialists, epidemiologists, nutritionists, infection control practitioners, public health officials, social scientists, family medicine physicians, pharmacists, microbiologists, and infectious disease physicians. A diverse group of organizations and agencies were represented at the workshop, including universities, regional geriatric programs, geriatric research units, long-term care research networks, acute care hospitals, long-term care facilities, home care nursing associations, provincial hospital associations, national nursing and geriatric nursing associations, health ministries, and pharmaceutical companies.

The workshop began with speakers from each of the four research disciplines broadly describing key issues in their respective discipline. Issues presented included immunologic changes associated with aging and nutritional strategies aimed at reversing immune senescence [7]; the challenge of conducting clinical research with older adults in long-term care facilities, including the difficulty in diagnosing infection and the limited availability of data in this area [8]; providing health services to older adults, including the limitations of administrative database research on current risk adjustments and the choice of outcome measures in this population [9]; and a presentation of a framework on how broader determinants of health may be examined with respect to infectious diseases in older adults [10].

The 54 participants were assigned to five groups and given instructions on how to frame research questions for both quantitative and qualitative research questions $[11,12]$. Specifically, the statement of study population, comparison, outcomes in a quantitative question versus the open-ended, hypothesis-generating nature of qualitative questions was emphasized. Each group included 
individuals from different research disciplines and stakeholders from each of the three different healthcare settings (long term, acute care and community). Participants in each group, assisted by a facilitator, discussed research priorities for each healthcare setting (acute care, community, long-term care). Five to ten research questions were identified for each setting. The questions generated from each group were collated. The facilitators taped all sessions and took written notes.

Following each session, participants reviewed the research questions generated and ranked the ten most important questions by assigning a numeric value from " 1 " to "10" for each question. The rankings were summarized by the Q - sort methodology, in which the numerical values for the ranking were inverted so that questions with the lowest priority ("1") were given the numeric value of "10", and vice versa [13]. Then for each question, the assigned numeric values were summed across all participants' lists. During the final half day of the workshop, the list of ranked research questions was presented to the entire group for discussion. This allowed participants to review and change rankings and ensure there was broad consensus about the results. Questions which were similar were amalgamated.

\section{Results and Discussion}

The research questions developed through this process are listed in order of priority sorted by healthcare setting (Tables 1 to 3). The research questions proposed by the workshop participants were diverse. They ranged from risk factors and outcomes for different infections, the impact of infections on quality of life, to the effect of nutrition on infection and the role of alternative and complementary medicine in treating infections (Tables $1,2,3)$. The questions related predominantly to health services, clinical sciences, and epidemiology/broader determinants. Health service issues participants deemed to be important included barriers to immunization, prolongation of hospital length of stay by infection, use of care paths for managing infections, the impact of infection on quality of life, and decision-making in determining the site of care for individuals with infections. Clinical questions included risk factor assessment for infection, the effectiveness of preventative strategies, and technology evaluation, such as the utility of videos in swallowing assessement. Epidemiologic issues included the challenge of achieving a better understanding of respiratory infections in the community and determining the prevalence of colonization with multi-resistant bacteria. These questions are of direct relevance to researchers in a variety of fields including primary care, nursing, nutritional science, public health, health services, gerontology, geriatrics, infection control, and infectious diseases.
Table I: Research questions for the acute care setting in descending order of priority.

I. In frail older adults, does the use of a critical care pathway for management of pneumonia reduce hospital stay and improve health-related quality of life?

2. For older adults over the age of 85 years, does admission to an intensive care unit for sepsis reduce mortality?

3. In hospitalized older adults, what are the modifiable risk factors for nosocomial pneumonia?

4. What are the barriers to implementation of a hospital-based pneumococcal vaccination program for older adults?

5. In older adults admitted to hospital with respiratory symptoms, how common is infection with influenza?

6. Does a computer-based learning intervention improve nurses' knowledge about infection control precautions for hospitalized older adults?

7. Compared to intravenous therapy, are highly bioavailable oral antibiotics effective in treating older adults admitted to hospital with pneumonia?

8. In older adults with nosocomial pneumonia, what are the most important predictors of excess length of stay?

9. Compared to usual care, do oral feeding programs for hospitalized older adults lead to earlier discharge?

10. What is the effectiveness of nursing-based strategies to reduce infection in hospitalized high risk older adults?

II. In hospitalized older adults, do swallowing evaluations and diet modifications reduce pneumonia and other respiratory infections?

12. Does early mobilizing of hospitalized older adults reduce the overall infection rate?

13. Amongst older adults admitted to hospital from nursing homes, what is the prevalence of colonization with multi-resistant bacteria and how common is transmission of these multi-resistant bacteria to other hospitalized patients?

14. In older adults, does early discharge from hospital reduce the risk for infection?

15. For older adults admitted to hospital with nursing home acquired pneumonia, does the addition of empiric anti-pseudomonal antibiotic coverage to standard therapy reduce mortality?

16. For specific nosocomial infections, are older adults treated with a longer duration of antibiotics than younger patients?

17. For older adults started on antibiotic therapy, does concurrent administration of an oral Lactobacillus preparation decrease the rate of Clostridium difficile-associated diarrhea?

18. In older adults hospitalized for pneumonia, what is the natural history of functional status?

19. Does a care path for urinary care reduce duration and frequency of urinary catherization in older hospitalized patients?

20. In older adults, does the administration of supplemental oxygen during joint replacement surgery decrease the risk of infection?

The question of whether a critical pathway for frail older adults with pneumonia can reduce length of hospital stay and improve quality of life received the highest priority ranking for the acute care setting. The Capital Study, a randomized trial for treatment of community acquired pneumonia using a clinical pathway, demonstrated that 
Table 2: Research questions for the community setting in descending order of priority.

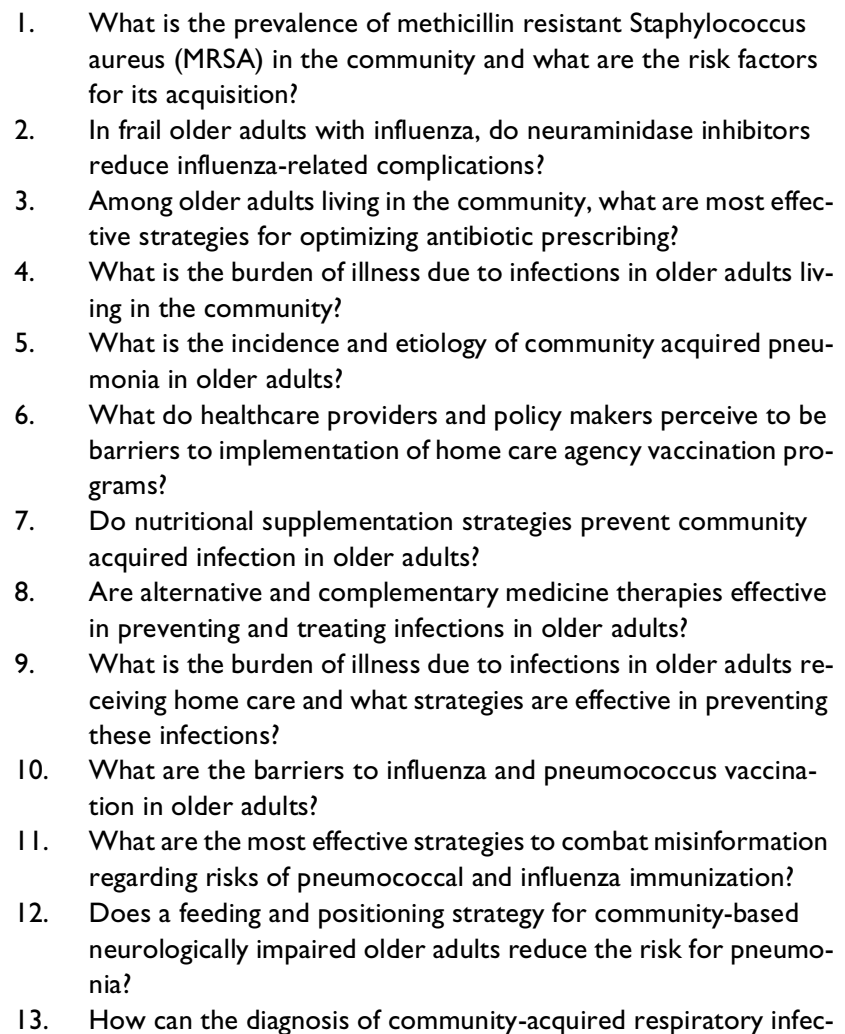

13. How can the diagnosis of commun
tions be improved in older adults?

patients managed using the pathway had a reduced stay in hospital and a quality of life equivalent to patients managed with usual care [14]. Whether a critical pathway, in addition to reducing time in hospital, leads to improved quality of life in patients over the age of 85 is unknown however. Ranked second for the acute care setting was the issue of whether admission of older adults over the age of 85 to intensive care for sepsis actually reduces mortality. This question obviously has important implications for patients, their families, and clinicians. Although reasonable rates of long-term survival in critically ill elderly patients requiring intensive care has been demonstrated $[15,16]$, outcomes of intensive care for patients 85 years and older specifically for sepsis have not been assessed. For the community setting, determining the prevalence of MRSA in the community received the highest priority. Addressing this question can potentially provide information about transmission patterns of MRSA as well as the need for empiric vancomycin therapy in older adults with presumed community-acquired Staphylococcus aureus infection. In Canada, true community acquired MRSA has been documented in the
First Nations population [17], but little is known about the prevalence in the general population. The second question prioritized for the community setting asks whether neuraminidase inhibitors, new anti-viral agents, reduce complications of influenza in older adults in the community. Although these agents reduce duration of symptoms for influenza, they have not demonstrated clear benefit in reducing complications such as hospitalization or death in the population at highest risk: older adults $[18,19]$. In the long-term care setting, the question which received the highest priority addressed factors associated with transfer to acute care hospital. Fried and colleagues found that only tachypnea and evaluation in the evening were associated with hospital versus long-term care facility evaluation and initial treatment [20]. However, their study long-term care facility was characterized by extensive physician involvement, potentially limiting the generalizability of the findings as stated by the authors. The second question prioritized for this setting asked whether institutional factors can help reduce the spread of antibiotic resistance. Factors of potential importance may include staffing, use of handwashing, use of anti-bacterial soaps, or the availability of sinks. Although $\mathrm{Li}$ and colleagues found an association between staffing levels and outbreaks in nursing homes in New York State [21], there has been no data addressing the effect of such variables on antibiotic resistance.

Not surprisingly, the research questions developed in this workshop closely reflected the ideas, experience, and agenda of the participants. To maintain feasibility, the majority of participants were from southern Ontario, limiting the choice of participants. These factors may have led to the high rank accorded to determining the prevalence of methicillin resistant Staphylococccus aureus (MRSA) in the community, likely a reflection of participants' interest in infection control as well as of the particularly high prevalence of MRSA in southern Ontario. Albeit infrequently, individuals with MRSA with no obvious risk factors (such as previous hospitalization) are being recognized in southern Ontario and concern about this likely led to the high rank for MRSA. Another limitation was that there were few basic scientists represented at the workshop. This lead to a list of clinically oriented questions with few basic research questions. However, despite the limitations imposed by our sample, we believe that the format used for this workshop can serve as a model for other research groups who wish to generate and prioritize research questions. Recently, research priorities have been developed in such diverse areas including critical care, physical activity and health among people with disabilities, and in emergency medical services for children [22-24]. Research agendas need to be comprehensive and cover the continuum of health- 
Table 3: Research questions for the long-term care facility setting in descending order of priority.

\footnotetext{
I. What factors are associated with the decision to transfer residents of long-term care facilities with fever or suspected infection to acute care hospitals?

2. What institutional strategies are effective in preventing infection in residents of long-term care facilities?

3. Does early nutritional intervention reduce severity or duration of infection?

4. Does a clinical pathway for management of nursing home acquired pneumonia improve outcomes and reduce cost?

5. What is the impact of MRSA on the quality of life in older adults who reside in long-term care facilities?

6. In the emergency room, what factors are associated with the decision to admit older adults with nursing home acquired pneumonia?

7. What is the most effective strategy for reducing inappropriate antibiotic prescribing?

8. What is the diagnostic accuracy of definition of infection in residents of long-term care facilities?

9. What quality of life outcomes resulting from infection are important from the perspective of staff, relatives and family?

10. What are the attitudes, perceptions, and beliefs of long-term care facility residents, healthcare providers, families regarding on-site treatment of infection in the long-term care facility?

II. In long-term care facilities, do isolation precautions reduce transmission of antibiotic resistant bacteria?

12. In residents of long-term care facilities, does eradication therapy for MRSA reduce transmission of this organism?

13. In residents of long-term care facilities with acute delirium and bacteriuria, do antibiotics improve mental status?
}

care settings. Our experience suggests that bringing together a multi-disciplinary group of researchers to frame and prioritize research questions about aging is feasible, and that participants valued the opinions of people working in other areas. In fact, the workshop has resulted in several multi-disciplinary collaborative partnerships among the participants. We also feel that the resultant list of research questions will help not only the workshop participants but also other researchers focus their interest in infections among older adults.

In order to disseminate the list of prioritized research questions, we plan to forward the research questions to Canadian geriatric and gerontologic research units as well as infectious disease research units which can distribute the results of the workshop to their members and post them on relevant web sites. We also will forward our findings to local provincial public health units, infection control practitioner associations, physician groups, long-term care groups, provincial funding agencies and advisory councils on aging.

\section{Conclusions}

The questions are of direct relevance to researchers in a wide variety of fields primary care, nursing, nutritional science, public health, health services, gerontology, geriatrics, infection control, and infectious diseases. Our experience suggests that bringing together a multidisciplinary group of researchers to frame and prioritize research questions about aging is feasible, and that participants valued the opinions of people working in other areas.

\section{Additional material}

\section{List of attendants for the Tri-council Workshop - April 13th and April 14th 2000, Hamilton, Ontario, Canada.}

List of names, titles, and corresponding institutions of Workshop participants

List of participants.doc

[http://www.biomedcentral.com/content/supplementary/14712318-1-1-s2.doc]

\section{Acknowledgments}

We would like to thank the 54 participants in this workshop (see Additional file 2: List of participants.doc), and Lorraine Moss for her help in organizing this workshop. This workshop was funded by the Canadian Institutes of Health Research.

\section{References}

I. Canadian Institute for Health Information: Health Report.1997

2. NACA: National Advisory Council of Aging. Health Canada

3. Kerr HD, Byrd JC: Nursing home patients transferred by ambulance to the emergency department. J Am Geriatr Soc I991, 39:132-136

4. Irvine PW, Van Buren N, Crossley K: Causes for hospitalization of nursing home residents: the role of infection. J Am Geriatr Soc 1984, 32:103-107

5. Bergman H, Clarfield AM: Appropriateness of patient transfer from a nursing home to an acute-care hospital: a study of emergency room visits and hospital admissions J Am Geriatr Soc I991, 39:। |64-| I68

6. Loeb M, McGeer A, McArthur M, Walter S, Simor AE: Risk factors for pneumonia and other lower respiratory tract infections in elderly residents of long-term care facilities. Arch Intern Med 1999, 1 59:2058-2064

7. High KP: Micronutrient supplementation and immune function in the elderly. Clin Infect Dis 1999, 28:717-22

8. Nicolle LE: Nursing home dilemmas. Infect Control Hosp Epidemiol 1997, I 8:806-808

9. BC Williams, Fries BE, Mehr DR: Patterns and determinants of health services use and mortality after VA nursing home care. J Aging Health 1996, 8:280-301

10. Evans ], Stoddart GL: Producing health, consuming health care. Soc Sci Med 1990, 3 I: 1347-1363

II. Counsell C: Formulating questions and locating primary studies for inclusion in systematic reviews. Ann Intern Med 1997, 1 27:380-387

12. Maxwell JA: Research questions: what you want to understand Chapter 4. In: Qualitative research design: an interactive approach. London, Sage Publications 199649-62

13. Sachs J: Using a small sample $\mathbf{Q}$ sort to identify item groups. Psychological Reports 2000, 86:287-294 
14. Marrie TJ, Lau CY, Wheeler SL, Wong CJ, Vandervoort MK, Feagan BG: A controlled trial of a critical pathway for treatment of community-acquired pneumonia. CAPITAL Study Investigators. Community-Acquired Pneumonia Intervention Trial Assessing Levofloxacin. JAMA 2000, 283:749-755

15. Chelluri L L, Pinsky MR MR, Donahue MP, Grenvik A A: Long-term outcome of critically ill elderly patients requiring intensive care. JAMA 1993, 269:3119-3123

16. Montuclard L L, Garrouste-Orgeas M, Timsit JF, Misset B, De Jonghe $B$, Carlet J: Outcome, functional autonomy, and quality of life of elderly patients with a long-term intensive care unit stay. Crit Care Med 2000, 28:3389-3395

17. Embil J, Ramotar K, Romance L, Alfa M, Conly J, Cronk S, Taylor G, Sutherland B, Louie T, Henderson E, Nicolle LE: Methicillin-resistant Staphylococcus aureus in tertiary care institutions on the Canadian prairies 1990-1992. Infect Control Hosp Epidemiol | 994, | 5:646-5|

18. Treanor JJ, Hayden FG, Vrooman PS, Barbarash R, Bettis R, Riff D, Singh S, Kinnersley N, Ward P, Mills RG: Efficacy and safety of the oral neuraminidase inhibitor oseltamivir in treating acute influenza: a randomized controlled trial. US Oral Neuraminidase Study Group JAMA 2000, 283:1016-24

19. Monto AS AS, Robinson DP, Herlocher ML, Hinson JM Jr, Elliott MJ, Crisp A: Zanamivir in the prevention of influenza among healthy adults: a randomized controlled trial. JAMA 1999, 282:3I-5

20. Fried TR, Muriel RG, Lipsitz LA: Whether to tansfer? Factors associated with hospitalization and outcome of elderly longterm care patients with pneumonia . J Gen Intern Med 1995, 10:246-250

21. Li J, Birkhead GS, Strogatz DS, Coles FB: Impact of institution size, staffing patterns, and infection control practices on communicable disease outbreaks in New York State nursing homes Am J Epidemiol 1996, 143: 1042-1049

22. Vella K, Goldfrad C, Rowan K, Bion J, Black N: Use of consensus development to establish national research priorities in critical care. BMJ 2000, 320:976-980

23. Cooper RA, Quatrano LA, Axelson PW, Harlan W, Stineman M, Franklin B, Krause JS, Bach J, Chambers H, Chao EY, Alexander M, Painter P: Research on physical activity and health among people with disabilities: a consensus statement. J Rehabil Res Dev 1999, 36:142-154

24. Seidel JS JS, Henderson D, Tittle S, Jaffe DM, Spaite D, Dean JM, M Gausche, Lewis RJ, Cooper A, Zaritsky A, Espisito T, Maederis D: Priorities for research in emergency medical services for children: results of a consensus conference. Ann Emerg Med 1999, 33:206-210

\section{Pre-publication history}

The pre-publication history for this paper can be accessed here:

http://www.biomedcentral.com/content/backmatter/

1471-2318-1-1-b1.pdf

Publish with BioMed Central and every scientist can read your work free of charge

"BioMedcentral will be the most significant development for disseminating the results of biomedical research in our lifetime."

Paul Nurse, Director-General, Imperial Cancer Research Fund

Publish with BMC and your research papers will be:

- available free of charge to the entire biomedical community

- peer reviewed and published immediately upon acceptance

- cited in PubMed and archived on PubMed Central

- yours - you keep the copyright

Submit your manuscript here:

http://www.biomedcentral.com/manuscript/
BiolMedcentral.com editorial@biomedcentral.com 